\title{
Violência contra a pessoa idosa: análise de aspectos da atenção de saúde mental em cinco capitais brasileiras
}

\author{
Violence against the elderly: analysis of aspects \\ of mental health care in five Brazilian cities
}

Fabiana Castelo Valadares ${ }^{1}$

Edinilsa Ramos deSouza ${ }^{1}$

${ }^{1}$ Centro Latino-Americano deEstudos deViolência eSaúdeJorgeCareli, Escola Nacional deSaúde Pública Sergio Arouca, Fundação O swaldo Cruz. Av. Brasil $4.036,7^{\circ}$ andar/ sala 700. 21040-361 Rio deJaneiro RJ.

fabianacastelo@yahoo.com.br
Abstract Thisisa research on the aspects of mental health care offered to elderly victims of violence in the cities of Curitiba, Rio de Janeiro, Brasília, Recife and M anaus. It was used the triangulation method that relied on the analysis of secondary data, information from questionnaires and individual and group interviews, defining itself as a target audience of this study, professionals and managers in mental health care. It was investigated the line of practices developed in services regarding policies governing mental health attention and addressed to the elderly who are victims of violence. Among the findings are: the disparity in records between elderly care hospitals $(22.3 \%)$ and outpatient $(0.4 \%)$, lack of information on the care of elderly victims of violence; and the incipient perception of professionals regarding the violence as a health intervention. In conclusion, there is a need for greater public investments: in improving the quality of information; the adequacy of the physical structure of services; the training/qualification of professionals to attend for specific elder health issues; under the aspect of violence, it was observed the need to adopt actions of prevention and attention of the most frequent disorders in the elderly. Key words Elder, Violence, Mental health and psychosocial
Resumo Trata-se de investigação sobre aspectos da atenção à saúde mental ofertada à pessoa idosa vítima de violência nos municípios de Curitiba, Rio de Janeiro, Brasília, Recifee $M$ anaus. Foi utilizada a abordagem de triangulação de métodos a partir da análise de dados secundários, informações de questionários e entrevistas individuais e em grupo, definindo-se como público-alvo profissionais e gestores da atenção à saúde mental. Investigou-se a consonância das práticas desenvolvidas nos serviços em relação às políticas que re gem a atenção à saúde mental e as dirigidas às pessoas idosas vítimas de violência. Entre os resultados destacam-se: a disparidade dos registros de atendimento de idosos entre as unidades hospitalares $(22,3 \%)$ e extra-hospitalares $(0,4 \%)$; a ausência de informações sobreo atendimento deidosos vítimas de violência; e a incipiente percepção dos profissionais quanto à violência como objeto de intervenção da saúde. Conclui-se pela necessidade de maiores investimentos públicos: na meIhoria da qualidade da informação; na adequação da estrutura física dos serviços; na capacitação/ habilitação dos profissionais para atenderem às especificidades de saúde do idoso. No aspecto da violência, observou-se a necessidade da adoção de ações de preven ção e atenção dos transtornos mais frequentes em idosos.

Palavras-chave Idoso, Violência, Saúde mental, Atenção psicossocial 
Introdução

O Brasil vive o fenômeno do envelhecimento de sua população - a expectativa de vida ao nascer mais que dobrou do início do século XX até os dias atuais ${ }^{1}$. Segundo estimativas do Instituto Brasileiro de Geografia e Estatística (IBGE)², em 2009 havia cerca de 19 milhões de idosos no país, o que representava aproximadamente $10 \%$ do total da população nacional, sendo mantida uma ten dência de crescimento deste grupo etário para os próximos anos. Esse acelerado crescimento da população idosa brasileira vem exigindo a construção de estratégias e políticas visando à promoção do envel hecimento saudável e a garantia dos direitos humanos, uma vez queo envel hecimento da população torna mais visíveis problemáticas antes consideradas "silenciosas", como o fenômeno da violência contra a pessoa idosa.

Segundo a Organização Mundial da Saúde $(\mathrm{OMS})^{3}$, a violência contra a pessoa idosa édefinida como: "A ções ou omissões cometidas uma vez ou muitas vezes, prejudicando a integridadefísica eemocional da pessoa idosa, impedindo o desempenho de seu papel social." No campo da saúde essa violência é registrada como maus-tratos, e seus impactossão representados no capítulo "Causas externas" da Classificação Internacional de Doenças (CID-10), em que são incluídos eventos não naturais que levam à morte ou provocam lesões e traumas. Segundo dados do Ministério da Saúde ${ }^{4}$ no ano de 2007, 18.946 idosos morreram por causas externas no Brasil, representando a sétima causa de morte da população acima de 60 anos. Nesse mesmo ano, houve cerca de 125.000 hospitalizações de idosos por essa causa.

Estudos demonstram quea maioria das queixas de violência contra idosos refere-se à violência praticada por parentes 5 . Noventa por cento dos casos de violência contra esse grupo ocorrem no interior dos lares; 2/3 dos agressores são filhos homens, noras, genrosecônjuges, eháuma forte associação nos casos em que o agressor físico e emocional usa drogas ${ }^{6,7}$. Contribuem para a maior vulnerabilidade os seguintes fatores: 0 agressor viver na mesma casa que a vítima; existirem relações dedependência financeira entrepais efilhos; 0 ambiente de pouca comunicação, pouco afeto e vínculos frouxos na família; o isolamento social da família e da pessoa idosa; haver história de violência na família; o cuidador ter sido vítima de violência doméstica; e a presença de qualquer tipo de sofrimento mental ou psiquiátrico ${ }^{8}$. Em relação à violência cometida contra idosos, estudos ${ }^{8,9}$ mostram a dificuldade das vítimas de revelarem os maus-tratos, seja por constrangimento, seja por temor a punições e retaliações de seus agressores.

0 fato de a presença de doenças mentais em agressores estar relacionada aos fatores que contribuem para a maior vulnerabilidade dos idosos ${ }^{10-14}$ convoca a rede de atenção à saúde mental ao enfrentamento e prevenção da violência. Os estudos desses autores revelam que $50 \%$ dos abusadores demonstram problemas com bebida alcoólica; que agressores físicos e emocionais usam álcool e drogas numa proporção três vezes maior que não abusadores; e ainda que a presença de depressão ou outros sintomas psiquiátricos está associada à transformação do cuidador em maltratante e abusador. No Brasil, a atual política de atenção orientada pela reforma psiquiátrica estabelece "a atuação no território ea clínica ampliada" como norte de ação ${ }^{15,16}$. Nessa perspectiva, 0 envolvimento das famílias e da comunidadecomo atores no processo detratamento do doentemental descortina relações complexas, em que a violência podeestar vigentee exigindo a intervenção da rede de saúde mental. No entanto, o idoso vítima de violência também éaquelequeapresenta quadro de doen ça mental, na maioria das vezes em estágio residual e, portanto, também integrante do grupo demaior vulnerabilidadeà violência por ser dependente de seus cuidadores. Estudos comprovam as influências da violência na irrupção de transtornos mentais, evidenciando a compreensão deque processos violentos deterioram a qualidade de vida ${ }^{17}$. Nesse sentido, diversos autore ${ }^{18-21}$ têm ressaltado a associação da violência com o surgimento de quadros psiquiátricos, principalmente a depressão. Entretanto, a maior parte dos estudos relacionados refere-se a mulheres, crianças e adolescentes vítimas de violência intrafamiliar ecomunitária, sendo encontrados poucos registros de estudos semelhantes enfocando a população idosa22.

Desde 1994, o Brasil vem desenvolvendo legislações direcionadas à população idosa, enfatizando as especificidades da atenção à saúde e inclusão social na Política Nacional do I doso ${ }^{23}$ eno Estatuto do Idoso 24 ; ea preocupação com o problema da violência na Política Nacional deSaúde do Idoso ${ }^{25}$, na Política Nacional de Redução da M orbimortalidade por Acidentes e Violências ${ }^{26} \mathrm{e}$ no Pacto pela Saúde ${ }^{27}$. Estas políticas preconizam a atenção integral à saúde dos idosos em todos os níveis de assistência, a ressocial ização e integração social, familiar ecomunitária, a capacitação de recursos humanos nas áreas mais diretamente relacionadas aos idosos, e o apoio a 
estudos e pesquisas. Em relação à aten ção à pessoa idosa vítima de violência, estabelecem sua ocorrência em todos os dispositivos da rede de saúde deforma integrada esensível às vulnerabilidades mais comuns a esse público.

Tomando o conjunto das políticas citadas como parâmetros norteadores, este artigo apresenta a avaliação da atenção a idosos vítimas de violênciana rede desaúdemental decinco capitais nacionais. Buscou-se investigar, por meio de indicadores avaliativos, a qualidade da atenção ofertada nos diferentes tipos de serviços abordados, relacionada aos números de atendimentos e qualidade da informação, estrutura física e de pessoal apropriadas e sua organização e adoção de medidas preventivas. Além dos indicadores relacionados, buscou-se investigar a percepção dos profissionais anteos casos deidosos vítimas deviolência expressos nas sínteses dos atendimentos.

\section{M etodologia}

Este trabalho é parte de uma pesquisa multicêntrica cujo objetivo foi avaliar 0 atendimento de sistemas locais de saúde aos agravos provocados por acidentes eviolências contra pessoas idosas. Foi desenvolvida em 2007 e 2008 em cinco capitais brasileiras das distintas regiões do país: M anaus, Recife, Braślia, Rio de Janeiro eCuriti$\mathrm{ba}^{28}$. A pesquisa original, de cunho exploratório, triangulou métodos quantitativo e qualitativo ${ }^{29}$. 0 presente artigo realiza a análise dos dados re lativos às unidades de saúde mental envolvidas na referida investigação.

A abordagem quantitativa da primeira fase do estudo consistiu na aplicação de um questio- nário com perguntas fechadas e abertas, que caracterizou as unidades de saúde mental que efetivamente atendiam a pessoas idosas vítimas de violência. A segunda fase visou qualificar esse atendimento aplicando-se um questionário específico a cada um dos distintos serviços/unidades de saúde mental, versando sobre o número deatendimentos, fluxo, estrutura física, composição da equipe, atividades desenvolvidas para a promoção da saúde do idoso e prevenção à violência, dentre outras questões. Trabalhou-se com uma amostra de conveniência que incluiu um Centro de Atenção Psicossocial (CAPS) por área de planejamento da saúde de cada município estudado (área programática, distrito sanitário ou outra). Assim, a amostra contou com 25 dos 46 CAPS existentes em 2009 na rede local dessas cidades e 12 dos 30 hospitais psiquiátricos em funcionamento nesse mesmo ano. Além deles, o estudo incluiu um ambulatório especializado e um serviço de reabilitação psicossocial, totalizando 39 serviços distribuídos em distintos tipos, como mostra a Tabela 1.

Em termos qualitativos, foram aplicadas 28 entrevistas semiestruturadas. Inicialmente, em cada município obteve-se o depoimento dos gestores municipais da área técnica; na segunda fase foram entrevistados coordenadores e psicólogos dos CAPS, bem como diretores, assistentes sociais e psiquiatras de hospitais psiquiátricos. Foi entrevistado um profissional de cada uma dessas categorias em cada uma das capitais. As entrevistas foram realizadas com base em quatro roteirossemiestruturados contendo entre 10 e 11 questões, direcionados segundo a especificidade do cargo do entrevistado, sendo um roteiro para os coordenadores eoutro para psicólogos dos CAPS;

Tabela 1. Distribuição dos serviços de saúde mental estudados por município.

\begin{tabular}{lccccccc}
\hline \multirow{2}{*}{ Municípios } & \multicolumn{7}{c}{ Tipos de serviços } \\
\cline { 2 - 8 } & $\begin{array}{c}\text { Ambulatório } \\
\text { especializado }\end{array}$ & $\begin{array}{c}\text { CAPS } \\
\text { II }\end{array}$ & $\begin{array}{c}\text { CAPS } \\
\text { III }\end{array}$ & $\begin{array}{c}\text { CAPS } \\
\text { ad }\end{array}$ & $\begin{array}{c}\text { Hospital } \\
\text { psiquiátrico } \\
\text { estadual }\end{array}$ & $\begin{array}{c}\text { Hospital } \\
\text { psiquiátrico } \\
\text { conveniado }\end{array}$ & Reabilitação \\
\hline Manaus & 0 & 0 & 1 & 0 & 1 & 0 & 0 \\
Recife & 0 & 5 & 1 & 3 & 2 & 4 & 0 \\
Brasília & 0 & 1 & 0 & 0 & 0 & 0 & 0 \\
Rio de Janeiro & 1 & 8 & 0 & 0 & 1 & 0 & 1 \\
Curitiba & 0 & 3 & 0 & 3 & 0 & 4 & 0 \\
Total & 1 & 17 & 2 & 6 & 4 & 8 & 1 \\
\hline
\end{tabular}

N ota: 0 serviço de reabilitação não foi considerado neste artigo. 
um para os diretores e outro para assistentes sociais e psiquiatras dos hospitais psiquiátricos.

Os questionários, respondidos por responsáveis designados pelos serviços, foram processados em um banco de dados e analisados em termos absolutos e relativos. A análise avaliativa do atendimento oferecido pelos serviços aos idosos vítimas de violência foi feita por meio de indicadores formulados com base nas políticas direcionadas à pessoa idosa e na política de saúde mental. Esses indicadores foram construídos e validados por meio do método de consenso, técnica do Grupo Nominal, segundo o qual foram realizadas reuniões altamente estruturadas com especial istas (sendo uma em cada capital) com o objetivo de coletar informações e viabilizar, por meio deavaliações individuais, discussão em grupo eposterior reavaliação da série deitensequestões propostas como indicadores ${ }^{30,31}$. A aplicação dos indicadores avaliativos - sendo 17 para os CAPS e sete para os hospitais psiquiátricos nas análises proporcionou uma visão ampliada e comparativa entre os municípios pesquisados.

As entrevistas foram gravadas e analisadas com o referencial da Análise deConteúdo segundo a modalidade temática adaptada da proposta de $\operatorname{Bardin}^{32}$, sendo conduzida basicamente por um recorte interpretativo que buscou compreender a construção social da realidade estudada, produzindo inferência do texto focal para o contexto social. N esse sentido, buscou-se investigar a percepção do atendimento ao idoso vítima de violência na rede de saúde mental. Todas as recomendações contidas na Portaria n 196/1996, do Conselho Nacional de Ética em Pesquisa, sobre seres humanos foram obedecidas.

Os resultados são apresentados em razão do nível da atenção ao qual o serviço se refere:

. Nível extra-hospitalar - compreende o ambulatório especializado e os CAPS.

- Nível hospitalar - reúne as informações apreendidas nos hospitais psiquiátricos.

\section{Resultadosediscussão}

\section{Atenção extra-hospitalar}

0 atendimento extra-hospitalar na saúde mental tem o CAPS como porta de entrada e organizador da rede de assistência. As unidades do tipo CAPS agregam as funções de pronto atendimento, acolhimento, suporte a crise, atenção dia e encaminhamento da demanda aos demais serviços da rede, desempenhando, portanto, funções de intermediária complexidade juntamente com os ambulatórios especializados ${ }^{15,33}$.

A Tabela 2 mostra o número de atendimentos realizados pelos CAPS em 2006. I nicialmente, chama a aten ção o fato de que dos 25 CAPS pesquisados 12 não informaram seus dados sobre os atendimentos em geral, 16 não o fizeram em relação ao atendimento de idosos e 21 não souberam informar sobre os casos de idosos vítimas de violência atendidos nos serviços. Tal ausência de informações também foi observada no ambulatório especializado, ondenão foram apresentadas quaisquer das informações solicitadas.

Para os 13 CAPS que informaram seus números de atendimento, a média foi de 3.153 para o total de atendimentos em 2006, de 16,4 atendimentos a pessoas idosas e de um idoso atendido em média por causa de violência. Observa-seque apenas quatro casos de idosos em situação de violência foram informados pelos CAPS de Curitiba. No entanto, é preciso relativizar essas informações, em razão da elevada quantidade de serviços que não responderam a essas questões, seja pela falta de registro sistematizado do seu atendimento, seja pela ausência de abordagem do tema.

A gestão das informações eo registro dosatendimentos realizados nos CAPS abordados foram avaliados por meio de indicadores. Um deles, que se refere à "Proporção de unidades/serviços que realizam o registro do atendimento aos acidentes/violências classificados deacordo com a CID-

Tabela 2. Distribuição dos atendimentos dos CAPS - 2006.

\begin{tabular}{|c|c|c|c|c|c|c|c|c|c|c|c|c|}
\hline \multirow[t]{2}{*}{ Atendimentos } & \multicolumn{2}{|c|}{$\begin{array}{c}\text { Manaus } \\
(\mathrm{N}=1)\end{array}$} & \multicolumn{2}{|c|}{$\begin{array}{l}\text { Recife } \\
(\mathrm{N}=9)\end{array}$} & \multicolumn{2}{|c|}{$\begin{array}{c}\text { Brasília } \\
(\mathrm{N}=1)\end{array}$} & \multicolumn{2}{|c|}{$\begin{array}{l}\text { Rio de Janeiro } \\
\quad(\mathrm{N}=8)\end{array}$} & \multicolumn{2}{|c|}{$\begin{array}{c}\text { Curitiba } \\
(N=6)\end{array}$} & \multicolumn{2}{|c|}{$\begin{array}{l}\text { Total } \\
(N=25)\end{array}$} \\
\hline & $\mathrm{N}$ & S/inf & $\mathrm{N}$ & S/inf & $\mathrm{N}$ & S/inf & $\mathrm{N}$ & S/inf & $\mathrm{N}$ & S/inf & $\mathrm{N}$ & S/inf \\
\hline Total & $\ldots$ & 1 & 2.802 & 5 & $\ldots$ & 1 & 34.498 & 3 & 3.688 & 2 & 40.988 & 12 \\
\hline Idosos & $\ldots$ & 1 & 94 & 6 & $\ldots$ & 1 & 17 & 6 & 37 & 2 & 148 & 16 \\
\hline Idosos por violência & $\ldots$ & 1 & $\ldots$ & 9 & $\ldots$ & 1 & 0 & 6 & 4 & 4 & 4 & 21 \\
\hline
\end{tabular}

0: informado pela unidade/serviço que não realizou o atendimento; ...: não informado pela unidade/serviço. 
10/DSM -IV e/ou quefazem análise sistemática dos registros", mostra que a unidade de Brasília, 70\% das de Recife, metade das do Rio de aneiro eapenas $40 \%$ dos CAPS de Curitiba afirmam atender pelo menos ao primeiro enunciado do indicador, ou seja, registram as informações segundo a CID10/DSM - IV. O CAPS de M anaus não respondeu a essa questão. É preciso ressaltar certa dissonância entre as informações fornecidas. Ao mesmo tempo que se diz que o atendimento é registrado, especificando-se os diagnósticos e a faixa etária dos pacientes, não se consegue informar os casos de violência diagnosticados nem se sabe dizer quantos idosos foram atendidos.

A precariedade dos registros é reforçada pelo fraco desempenho do outro indicador queavalia a "Proporção de unidades/serviços que realizam ações voltadas para a identificação de casos de idosos vítimas de acidentes e violência e/ou que notificam sistematicamente os casos de violência contra idosos para autoridade policial ou M inistério Público ou conselhosmunicipais eestaduais do idoso ou secretarias municipais eestaduais de Saúde ou Secretaria de Desenvolvimento Social". Com basenele, verifica-se que apenas $37,5 \%$ das unidades do Rio de Janeiro, 33\% das de Curitiba e $11 \%$ das de Recife cumprem essas diretrizes de diagnóstico e notificação dos casos. Os CAPS de $M$ anaus e Brasília não realizam nenhuma dessas duas orientações das políticas.

$\mathrm{Na}$ análise dos dados, também ressaltam as grandes disparidades entre as capitais pesquisadas quanto à qualidade da gestão das informações. N esse sentido, os indicadores que a avaliam mostram que os CAPS de Curitiba e do Rio de Janeiro apresentam os melhores resultados, enquanto os de Brasília e M anaus têm os piores desempenhos nesse item. Já os de Recife ficam em situação intermediária.

A maioria dos serviços pesquisados tem vinculação municipal eofereceatendimento deacordo com a lógica da territorialidade. Todos participam da rede de atenção à saúde mental de seus municípios, recebendo demanda espontânea e encaminhada, inclusive em relação a atendimentos de urgência, e realizando encaminhamentos dos casos aos demais serviços da rede de saúde e de assistência social. O CAPS estudado em Brasília informou não realizar atendimento de urgência. $O$ de $M$ anaus encaminha os idosos que necessitam de atenção de menor complexidade para a redeambulatorial da aten ção básica epara serviços especiais, como o Centro de Atenção Integral da M elhor Idade (Caimi), queé municipal e especializado na atenção à pessoa idosa.
No Rio de Janeiro, os CAPS informaram ter dificuldades para 0 encaminhamento dos pacientes à rede ambulatorial devido à superlotação dos serviços, fato que contribui para um crescentenúmero de pacientes nos CAPS. Em 2006, a média anual de atendimentos realizados nesses serviços foi de 6.899,6.

Em Manaus, Recife e Rio de Janeiro, foram relatadas dificuldades de encaminhamento de idosos às internações em hospitais gerais e psiquiátricos. Segundo os entrevistados, a indicação para internação é limitada e somente é feita mediante acompanhante.

Essefluxo do atendimento na redefoi avaliado pelo indicador queconsidera a "Proporção deunidades/serviços com mecanismos estabelecidos de referência e contrarreferência para casos que necessitam de internação e/ou que possuem integração com equipes do PSF visando à manutenção da capacidade funcional do idoso". Constatou-se que a unidade de Brasília ea de $M$ anaus, $83 \%$ das de Curitiba, $50 \%$ das do Rio de Janeiro e $22 \%$ das de Recife afirmam possuir tais mecanismos.

A infraestrutura física das unidades foi investigada a fim de verificar sua adequação às necessidades da pessoa idosa. Constatou-se que os acessos diferenciados para ambulâncias e para os idosos e portadores de deficiências físicas ainda são itens carentes de adaptação em todos os serviços. Em relação ao indicador "Proporção de unidades/serviços que possuem cadeiras de rodas, acesso diferenciado para idosos e/ ou portadores de necessidades especiais", os CAPS de Curitiba foram os únicos que registraram atendê-lo em $50 \%$ de suas unidades. Os demais municípios não pontuaram esse indicador.

0 ambulatório especializado do Rio de Janeiro informou possuir salas de recepção e espera, de arquivo e prontuário, de serviço social, bem como consultórios médico e psicológico, farmácia e acesso diferenciado para ambulância.

A adequação da estrutura física das unidades constitui um fator importante para a recepção e 0 acolhimento de idosos e portadores de necessidades especiais, ao se partir da compreensão de que cabem ao CAPS a clínicaampliadaeo envolvimento defamiliares na atenção ao doentemental. N esse sentido, a ausência de recursos e adequações estruturais reforça os outros estudos1, os quais indicam que os idosos encontram maiores dificuldades de acesso aos serviços de saúde em geral.

A constituição de equipes multiprofissionais capacitadas e especializadas na aten ção às necessidades mais comuns das pessoas idosas é uma diretriz das políticas de atenção à saúde desse 
grupo. Segundo os dados dos questionários, as categorias profissionais mais presentes nos CAPS e no ambulatório especializado são aquelas tradicionais da área. Por sua vez, a adequação das equipes de profissionais ao que recomendam as políticas foi avaliada por três indicadores. No primeiro, referenteà"Proporção deunidades que possuem profissionais com formação em gerontologia", constatou-sequeapenas dois CAPS (um em Curitiba e outro no Rio de Janeiro) informam possuir tal profissional em seu quadro. Em relação ao segundo indicador, "Proporção de unidades que realizam atividades de atendimento geriátrico e gerontológico", apenas um CAPS de Curitiba informou desenvolver tal atividade. Finalmente, o terceiro indicador, "Proporção de unidades cujos profissionais recebem capacitação para identificação e atendimento dos casos deidosos vítimas deacidentes eviolência", écumprido por apenas 33\% dos CAPS pesquisados em Curitiba.

A diversidade de ações realizadas nos CAPS foi investigada por meio de dez indicadores avaliativos, conforme se vê na Tabela 3.

Tabela 3. Indicadores avaliativos das ações desenvolvidas pelos CAPS, segundo as cidades pesquisadas.

\begin{tabular}{|c|c|c|c|c|c|}
\hline Indicadores & Manaus & Recife & Brasília & $\begin{array}{l}\text { Rio de } \\
\text { Janeiro }\end{array}$ & Curitiba \\
\hline $\begin{array}{l}\text { 1) Proporção de unidades/serviços que realizam } \\
\text { atividades de atendimento em oficinas terapêuticas, } \\
\text { atividades comunitárias enfocando a integração do } \\
\text { paciente na comunidade e sua inserção familiar e } \\
\text { social. }\end{array}$ & _ & $80 \%$ & $100 \%$ & $100 \%$ & $100 \%$ \\
\hline $\begin{array}{l}\text { 2) Proporção de unidades/serviços que realizam } \\
\text { atividades de atendimento grupal. }\end{array}$ & - & $66,7 \%$ & - & - & $100 \%$ \\
\hline 3) Atendimento de urgência. & $100 \%$ & $55,6 \%$ & $100 \%$ & $75 \%$ & $83,3 \%$ \\
\hline 4) Atendimento geriátrico e/ou gerontológico. & não & não & não & não & $16,7 \%$ \\
\hline 5) Visita domiciliar. & $100 \%$ & $100 \%$ & $100 \%$ & $100 \%$ & $83,3 \%$ \\
\hline $\begin{array}{l}\text { 6) Proporção de unidades/serviços que realizam busca } \\
\text { ativa para casos mais graves de idosos em situação de } \\
\text { violência quando há problemas de adesão ao } \\
\text { tratamento. }\end{array}$ & não & $77,8 \%$ & $100 \%$ & $75 \%$ & $83,3 \%$ \\
\hline $\begin{array}{l}\text { 7) Proporção de unidades/serviços que encaminham } \\
\text { pacientes para o Programa de Volta para Casa. }\end{array}$ & não & $77,8 \%$ & não & $75 \%$ & $50 \%$ \\
\hline $\begin{array}{l}\text { 8) Proporção de unidades/serviços que realizam ações } \\
\text { voltadas para o autocuidado; ações de promoção da } \\
\text { atividade física e alimentação saudável; ações para } \\
\text { redução de sequelas e incapacidades; ações voltadas } \\
\text { para a redução de danos; informações/cursos de } \\
\text { capacitação de cuidadores de idosos. }\end{array}$ & $60 \%$ & $80 \%$ & $60 \%$ & $58 \%$ & $67 \%$ \\
\hline $\begin{array}{l}\text { 9) Proporção de unidades/serviços que realizam ações } \\
\text { de estímulo à participação das pessoas idosas em grupos } \\
\text { da terceira idade, conselhos comunitários, clubes; } \\
\text { identificação e reconhecimento da rede de suporte } \\
\text { social para a pessoa idosa; informam sobre direitos dos } \\
\text { idosos. }\end{array}$ & $67 \%$ & $74 \%$ & $100 \%$ & $54 \%$ & $83 \%$ \\
\hline $\begin{array}{l}\text { 10) Proporção de unidades/serviços que oferecem uma } \\
\text { abordagem preventiva com aplicação de instrumentos } \\
\text { de avaliação e testes de triagem para detecção de } \\
\text { distúrbios cognitivos e/ou visuais e/ou mobilidade e/ou } \\
\text { audição e/ou depressão e/ou comprometimento da } \\
\text { funcionalidade e/ou distúrbios nutricionais e/ou } \\
\text { isolamento social e/ou que realizam ações de prevenção } \\
\text { de violências e de acidentes no domicílio. }\end{array}$ & $44 \%$ & $46 \%$ & $44 \%$ & $40 \%$ & $50 \%$ \\
\hline
\end{tabular}


Observa-se maior proporção de CAPS desenvolvendo atividades tradicionais como a busca de integração social do pacienteà comunidade e ao convívio familiar, o atendimento de urgência e a visita domiciliar. Em contraposição, indicadores mais especificamente direcionados às pessoas idosas, como a promoção do autocuidado (indicador 8) e o suporte social (indicador 9), estão menos pontuados.

Vale destacar o pior desempenho, em todas as cidades, dos indicadores avaliativos da abordagem preventiva aos transtornos mais comuns entre idosos e do atendimento geriátrico ou gerontológico. Observou-se que a abordagem preventiva da depressão, do isolamento social, do comprometimento da funcionalidade e dos distúrbios nutricionais érealizada em mais da metade dos 25 CAPS pesquisados. No entanto, menos da metade das unidades realiza abordagem preventiva relacionada a distúrbios cognitivos, visuais, de mobilidade e audição. Esse rastreamento é importante para a interven ção precoce e a minimização das sequelas e limitações na vida diáriae atende às diretrizes das políticas públicas dirigidas a essa população. Apesar de não se tratar de atribuições específicas da atenção em saúde mental, é imprescindível que os profissionais da área estejam sensíveis a essas questões visando ao encaminhamento às unidades especializadas.

$\mathrm{N}$ as entrevistas com os profissionais, a realização de atividades direcionadas à pessoa idosa foi pouco relatada, corroborando as informações prestadas nos questionários. As exceções foram no CAPS de M anaus, onde os idosos são remetidos ao Caimi, eno CAPS de Braślia, onde se desenvolve um trabalho específico com o público idoso que conta com a presença de acompanhantes terapêuticos. Deslandes et al. ${ }^{34}$ também encontraram semelhanteincipiência deações direcionadas às necessidades de saúde dos idosos. Os entrevistados imputaram essa ausência de especificidade à baixa demanda de idosos nos CAPS; entretanto, a precariedade de recursos, 0 excesso de demanda de pacientes em outras faixas etárias e a necessidade de se estabelecerem prioridades no cotidiano da atenção podem estar relacionados a essa interpretação.

Apesar dessa constatação, os depoentes re conhecem e destacam algumas necessidades do atendimento a esse público, como: (1) maior atenção na definição do diagnóstico, que pode mais frequentemente ser influenciado por outras patologias não psiquiátricas; (2) adaptação dos serviços em termos de acessibilidade; (3) cuidados especiais mais comumente desempenhados por um cuidador ou acompanhante; e (4) trabalho com as famílias.

Foi unânime a percepção das equipes técnicas quanto à ausência de serviços e profissionais habilitados para atender às necessidades mais comuns das pessoas idosas. A formação continuada e a adequação da rede de saúde foram as suas principais sugestões para melhorar a qualidade da atenção.

Pediu-se aos profissionais que relatassem casos atendidos de pessoas idosas vítimas de violência, objetivando identificar suas percepções sobrea atenção extra-hospitalar de saúde mental fornecida, as violências vividas, as estratégias de acolhimento e de encaminhamento. Um dos casos relatados é o de uma mulher idosa que se encontrava em situação de rua e por isso foi remetida ao CAPS de referência da área. Segundo 0 relato dos profissionais, essa senhora afirmava sofrer violência doméstica, sendo agredida pela filha, razão pela qual optou por residir na rua. A idosa manteve tratamento no CAPS mesmo permanecendo em situação de rua. No entanto, após um período, parou de frequentar o serviço, não sendo mais localizada. Segundo informações do serviço, ela foi procurada na residência dos familiares, mas não foi encontrada. 0 caso se encerra com o abandono do tratamento e o desaparecimento da paciente, não tendo sido relatado qualquer trabal ho de contato prévio com afamília em questão ou com a rede deapoio e proteção social.

Esse relato apresenta um traço comum entre as unidades extra-hospitalares pesquisadas em relação à não identificação da pessoa idosa como alvo do atendimento dos serviços de saúde mental. Para os entrevistados, a ausência de uma abordagem integral aos idosos nos serviços está associada à constatação de que são menos comuns os casos de idosos com quadro psiquiátrico agudo. Essa percepção opera, portanto, na limitação dos acolhimentos e logo numa menor investigação da complexidade que pode estar presente nas demandas com que esse grupo social chega à unidade. Com base nos questionários enas entrevistas realizadas, observou-se que a possibilidade de as violências provocarem impactos na saúde dos atendidos é ainda pouco investigada nos serviços. Observou-se também que os idosos são, muitas vezes, os principais cuidadores dos doentes mentais em tratamento nas unidades extra-hospitalares, o que os expõe a mai or vulnerabilidade. A pesar desta constatação, a atuação dos profissionais com relação às vítimas de violência ainda é pouco registrada. Estudos já apontaram para o fato de poucos pro- 
fissionais reconhecerem seu papel no tratamento, com a afirmativa de que não estão preparados para lidar com esse público ${ }^{35}$, o que é corroborado pelo relato de ausência de capacitação das equipes como demonstrado no indicador sobre o tema.

Ainda segundo o relato dos entrevistados, 0 acolhimento deidosos vítimas deviolência em unidades de saúde mental somente se justifica quando associado a um quadro psiquiátrico agudo de psicose, depressão grave e deficiência mental, por exemplo. Quando percebidas, as violências mais relatadas pelos entrevistados foram: a negligência e 0 abandono. Sendo diagnosticadas, o procedimento mais comum foi remeter os casosà justiça, pois ainda prevalece a compreensão de que esseé um problema da justiça ou de polícia e não um tema para ser abordado pelo setor saúde.

\section{Atenção hospitalar}

No contexto atual da política de saúde mental, as unidades que realizam a internação têm como função fornecer suporte e atendimento à crise. N esse modelo de atenção, a internação em hospital psiquiátrico éindicada como medida de proteção do doente, que requer cuidado intensivo, momentâneo e fora de seu meio familiar. Desse modo, recomenda-se internação psiquiátrica de curta duração, o que pressupõe alta rotatividade dos leitos e forte integração dessas unidades à rede de assistência extra-hospitalar ${ }^{15}$.

$A$ atenção à pessoa idosa vítima de violência que demanda cuidado intensivo de saúde mental foi investigada em 12 hospitais psiquiátricos, distribuídos em quatro das cinco capitais estudadas. 0 hospital psiquiátrico que atende a população de Brasília situa-se no Distrito Federal, portanto, fora da circunscrição da cidade. Mais uma vez, nota-sequeé el evado o número de unidades que não responderam às questões relativas ao seu atendimento, provavelmente devido à inexistência de registros sistemáticos e detal hados desses dados.

A Tabela 4 mostra que os idosos representam $10,4 \%$ dos atendidos no hospital de $\mathrm{Ma}$ naus; $38,6 \%$ dos atendidos nas quatro unidades deRecife; $17,9 \%$ dos queseinternaram no hospital do Rio de Janeiro; e 7,1\% de dois hospitais de Curitiba. No conjunto das cidades, os idosos representam a parcela de $22,3 \%$ do total das pessoas atendidas nos hospitais psiquiátricos que informaram esse dado, percentual significativamente superior ao $0,4 \%$ do total de idosos atendidos nos CAPS pesquisados.

A alarmante discrepância entre os números de atendimento hospitalar e extra-hospitalar merece destaque, uma vez que estudos ${ }^{36,37}$ já revelaram as precárias condições em que são tratados idosos internados por longos períodos em instituições psiquiátricas, e mesmo os limites da atenção psiquiátrica hospitalar para o tratamento do doente mental.

Todos os hospitais pesquisados compõem a rede de aten ção à saúdemental de seus municípios, cujo fluxo de atendimento está constituído pelo acolhimento à demanda espontânea ou encaminhada por outras unidades de saúde, via Central de Regulação de Vagas e/ou Leitos, em esquema de pronto atendimento ( 24 horas). Curitiba é a exceção, pois realiza atendimento somente mediante agendamento.

A investigação da adequação da estrutura física dos hospitais para 0 atendimento as necessidades mais comuns aos idosos indica que um hospital de Recife possui recursos tecnológicos de alta complexidade mais amplos que os recomendados a uma unidade psiquiátrica. Esta ins-

Tabela 4. Distribuição do número de atendimentos dos hospitais psiquiátricos

\begin{tabular}{|c|c|c|c|c|c|c|c|c|c|}
\hline \multirow[t]{2}{*}{ Atendimentos } & \multicolumn{2}{|c|}{$\begin{array}{c}\text { Manaus } \\
(\mathrm{N}=1)\end{array}$} & \multicolumn{2}{|c|}{$\begin{array}{l}\text { Recife } \\
(\mathrm{N}=6)\end{array}$} & \multicolumn{2}{|c|}{$\begin{array}{l}\text { Rio de Janeiro } \\
\qquad(N=1)\end{array}$} & \multicolumn{2}{|c|}{$\begin{array}{c}\text { Curitiba } \\
(\mathrm{N}=4)\end{array}$} & \multirow{2}{*}{$\begin{array}{c}\begin{array}{c}\text { Total } \\
(\mathrm{N}=12)\end{array} \\
\mathrm{N}\end{array}$} \\
\hline & $\mathrm{N}$ & S/inf & $\mathrm{N}$ & S/inf & $\mathrm{N}$ & S/inf & $\mathrm{N}$ & S/inf & \\
\hline Total & 31.572 & - & 26.780 & 2 & 11.881 & - & 580 & 2 & 70.813 \\
\hline Idosos & 3.285 & - & 10.325 & 2 & 2.130 & - & 41 & 2 & 15.781 \\
\hline Idosos por acidentes & $\ldots$ & 1 & $\ldots$ & 3 & $\ldots$ & 1 & 0 & 3 & 0 \\
\hline Idosos por violências & $\ldots$ & 1 & $\ldots$ & 3 & $\ldots$ & 1 & 0 & 3 & 0 \\
\hline $\begin{array}{l}\text { Internações } \\
\text { domiciliares de idosos }\end{array}$ & $\ldots$ & 1 & 0 & 3 & $\ldots$ & 1 & 0 & 3 & 0 \\
\hline
\end{tabular}


tituição éuma unidade deemergência psiquiátrica incluída em um hospital geral do município, 0 que justifica a presença dos recursos investigados. Os demais hospitais pesquisados não responderam a esta questão ou marcaram "não" para todos os itens.

A avaliação da atenção oferecida pelos hospitais psiquiátricos aos idosos vítimas de violência foi feita com base em seteindicadores, distribuídos em três blocos de análise: os quatro primeiros avaliam a gestão da informação; o quinto e o sexto analisam a adequação da equipe profissional; e o sétimo tenta captar a realização de atividades preventivas dos agravos mais comuns entre as pessoas idosas (Tabela 5).

Os indicadores relativos à gestão das informações apontam para um cenário favorável no conjunto das capitais pesquisadas. Entretanto, cabe destacar a incoerência desses resultados comparados a outros itens do questionário que demonstram a precariedade ou inexistência de registros em muitas das unidades pesquisadas, principalmenteos relacionadosao desdobramen- to por faixa etária, diagnóstico e notificação dos casos de violência.

Os hospitais pesquisados contam com a equipe mínima de profissionais recomendada pelas políticas que nortearam este estudo. 0 indicador "Proporção de unidades que possuem equipes mínimas para internação/assistência domiciliar (médico, enfermeiro, técnico/auxiliar de enfermagem, geriatra, profissional com formação em gerontologia)" demonstra que os hospitais de M anaus e Rio de Janeiro, $75 \%$ dos de Curitiba e $66,7 \%$ dos de Recife possuem pelo menos três dos seis profissionais exigidos pela política; no entanto, nenhum destes informou registros de atendimento domiciliar nos questionários aplicados. Dentre as categorias profissionais mais presentes, estão as que tradicionalmente compõem a assistência psiquiátrica.

Quanto à "Proporção de unidades que possuem profissional com formação em gerontologia", nota-se que apenas o hospital do Rio de Janeiro e um de Recife contam com essa categoria profissional em seu quadro; e apenas neste últi-

Tabela 5. Indicadores avaliativos da atenção oferecida nos hospitais psiquiátricos a idosos vítimas de violência, segundo as cidades pesquisadas.

\begin{tabular}{|c|c|c|c|c|}
\hline Indicadores & M anaus & Recife & $\begin{array}{c}\text { Rio de } \\
\text { Janeiro }\end{array}$ & Curitiba \\
\hline $\begin{array}{l}\text { 1) Proporção de unidades/serviços que possuem registros sobre os } \\
\text { atendimentos às pessoas em situação de violências/acidentes. }\end{array}$ & $100 \%$ & $100 \%$ & $100 \%$ & $75 \%$ \\
\hline $\begin{array}{l}\text { 2) Proporção de unidades/serviços que já ouviram falar e já leram } \\
\text { a PNRM AV, a Política Nacional de Saúde da Pessoa Idosa e o } \\
\text { Estatuto do Idoso (pelo menos um dos documentos). }\end{array}$ & $100 \%$ & $83 \%$ & $100 \%$ & $75 \%$ \\
\hline $\begin{array}{l}\text { 3) Proporção de unidades/serviços que realizam ações voltadas } \\
\text { para a identificação de casos de idosos vítimas de acidentes e } \\
\text { violência e/ou que enviam a notificação de casos de violência } \\
\text { contra idosos para autoridade policial ou M inistério Público ou } \\
\text { conselhos municipais e estaduais do idoso ou secretarias } \\
\text { municipais e estaduais de Saúde ou Secretaria de } \\
\text { Desenvolvimento Social. }\end{array}$ & $100 \%$ & $66,7 \%$ & $100 \%$ & $75 \%$ \\
\hline $\begin{array}{l}\text { 4) Proporção de unidades/serviços que realizam o registro dos } \\
\text { casos de idosos vítimas de acidentes e violência de acordo com a } \\
\text { CID-10 ou DSM -IV e/ou com pessoal capacitado para registro } \\
\text { sistemático da informação. }\end{array}$ & $100 \%$ & $66,6 \%$ & $100 \%$ & $100 \%$ \\
\hline $\begin{array}{l}\text { 5) Proporção de unidades que possuem equipes mínimas para } \\
\text { internação/assistência domiciliar. }\end{array}$ & - & - & $100 \%$ & - \\
\hline $\begin{array}{l}\text { 6) Proporção de unidades que possuem profissional com } \\
\text { formação em gerontologia. }\end{array}$ & - & $16,7 \%$ & $100 \%$ & - \\
\hline $\begin{array}{l}\text { 7) Proporção de unidades que oferecem abordagem preventiva } \\
\text { com aplicação de instrumentos de avaliação e testes de triagem } \\
\text { para detecção de distúrbios mais comuns em idosos. }\end{array}$ & - & $16,7 \%$ & $100 \%$ & $50 \%$ \\
\hline
\end{tabular}


mo município foi registrada também a presença de um médico geriatra atuando como tal.

A análise da"Proporção de unidades que oferecem abordagem preventiva com aplicação de instrumentos de avaliação etestes detriagem para detecção de distúrbios cognitivos, visuais, mobilidade, audição, depressão, comprometimento da funcionalidade, distúrbios nutricionais e isolamento social" constituiu um indicador que visava captar esforços do serviço para manter a qualidade de vida da pessoa idosa. Sua aplicação resultou pouco promissora. Verificou-se que apenas a unidade do Rio de Janeiro, $50 \%$ das de Curitiba esomente $16,7 \%$ das de Recife informaram realizar pelo menos quatro das oito abordagens recomendadas. As mais investigadas são as já tradicionais para a área psiquiátrica: de pressão, isolamento social, comprometimento da funcionalidade e distúrbios nutricionais.

O bjetivando compreender a atenção prestada aos idosos vítimas de violência que buscam o hospital psiquiátrico, solicitou-seà equipetécnica que relatasse casos de seu conhecimento. A título de ilustração, descreve-se aqui o caso de uma senhora que, mesmo com condições clínicas para a alta médica, permaneceu internada devido ao abandono de sua família. 0 profissional disse que ela já havia sido internada anteriormentena unidade. Para efetivar a alta, a equipea levou até sua casa, situada em uma área considerada "derisco" da cidade, eorientou seus familiares quanto ao uso da medicação. Contudo, pouco tempo depois a senhora retornou à internação, após ser de novo negligenciada. A equipe da unidade fez a denúncia ao M inistério Público e aguardava 0 seu pronunciamento sobre 0 caso, no momento do relato.

Esta situação parece ser comum nos outros hospitais pesquisados, reforçada por relatos de gestores quedisseram quea maior partedosidosos encontrados nas instituições psiquiátricas permanece em situação de abandono en egligência, por parte tanto de seus familiares quanto do poder público. Em tais situações, chama a atenção a pouca ou nenhuma intervenção dos profissionais diante de situações de violência e muitas vezes naturalizadas na própria estrutura hospitalar. Assim como ocorre nos CAPS, também prepondera entre os profissionais dos hospitais psiquiátricos 0 entendimento de que tais questões devem ser resolvidas na justiça ou por autoridades judiciais. Essa percepção da violência como um problema criminal e não de saúde pública, por parte dos profissionais da saúde, também foi evidenciada por Dahlberg e Krug ${ }^{38}$.
A isso acresce-se o fato de que a investigação e a identificação da violência contra os idosos são ainda muito pouco realizadas nas unidades de saúde mental, indicando um processo de naturalização das violênciastípicas dessa etapa da vida. M inayo eSouzåtambém identificaram esse processo e dizem que a violência cometida contra idosos pode ser compreendida a partir do abuso estrutural e societal. Segundo as autoras, as violências estrutural e societal referem-se a negligências das políticas públicas, dos serviços de saúde e das instituições - negligências e abandonos que dizem respeito à ausência ou privação de cuidados; desrespeito e atitudes de menosprezo correspondendo ao não reconhecimento; abusos verbais, emocionais e psicológicos que se referem às ofensas que diminuem a autoestima da pessoa; abusos físicos e abusos legal e financeiro. Tais categorias parecem ter sido ainda pouco assimiladas e incorporadas às práticas dos profissionais dos serviços de saúde mental.

\section{Conclusões}

A análise realizada mostra que a implementação das políticas relacionadas neste estudo na atenção à saúdemental, mais especificamente da atenção prestada às pessoas idosas vítimas de violência, ainda é bastante incipiente, provavelmente devido à novidade dos dois temas aqui abordados: a violência que provoca diferenciados distúrbios psíquicos e 0 atendimento a um grupo populacional envelhecido, cada vez mais crescente na realidade brasileira.

Do ponto de vista da atenção prestada pela redede serviços de saúdemental aqui investigada, percebe-se que tanto as unidades hospitalares quanto as extra-hospital ares vêm buscando cumprir as recomendações feitas nas políticas dirigidas à pessoa idosa. No entanto, em termos de estrutura física os hospitais parecem estar mais bem adaptados, possivelmente por se tratar de uma rede há muito consolidada, o que não significa que o hospital seja o melhor local para idosos vítimas de violência, uma vez queo risco de abandono enegligência, também nessas instituições, é bem maior que na atenção comunitária. Desse modo, os serviços CAPS, ainda em fase de implantação em algumas das cidades estudadas, necessitam ser apoiados no sentido de melhorar sua atuação em relação às questões aqui tratadas.

Para o conjunto dos indicadores aplicados aos CAPS pesquisados nas cinco capitais, Curitiba destaca-se como a que melhor vem desempe- 
nhando a implementação das políticas de saúde da pessoa idosa. Ao se compararem as capitais, Rio de Janeiro e Recife situam-se em posição intermediária, enquanto Brasília e M anaus encontram-se em pior colocação. Cabe lembrar que neste estudo foi abordada uma amostra de conveniência, não sepodendo generalizar os resultados aqui encontrados e devendo-se considerar a complexidade das distintas realidades sócio-históricas e da organização das redes dos municípios. Em relação ao atendimento hospitalar, o Rio de Janeiro se destaca como a capital que meIhor vem implementando as políticas avaliadas; no entanto, esses achados devem ser relativizados, tendo-se em vista que apenas um dos 15 hospitais existentes nesse município foi incluído no estudo. Em comparação com as demais capitais, $M$ anaus permanece em situação intermediária; já Recife e Curitiba encontram-se em pior colocação em relação ao processo de implementação das políticas avaliadas.

Este estudo limitou-se à análise do processo de implantação e implementação das políticas direcionadas à pessoa idosa vítima de violência. $N$ esse sentido, pontos importantes sobre a atenção da saúde mental a famílias que contam com idosos entre seus membros precisam ser mais bem explorados em futuras pesquisas.

Dentreos pontos críticos que mereceriam investimento público na área, destaca-se a necessidade de melhorar a qualidade da informação, principalmente quando abordado 0 atendimento de idosos vítimas de violência; de adequar a estrutura física dos serviços a fim de facilitar o acesso desse grupo; decapacitar/habilitar os profissionais para atenderem adequadamente às especificidades de saúde do idoso eno tema da violência; edeinvestir em ações de prevenção eatenção aos transtornos mais frequentes em idosos, o que pode promover qualidade de vida e reduzir as demandas de internação psiquiátrica e em hospitais gerais. A sensibilização dos gestores e trabalhadores da aten ção consiste, portanto, num ponto fundamental para viabilizar a implantação e a implementação adequadas das referidas políticas.
Colaboradores

FC Valadares e ER Souza trabalharam na concepção, redação e revisão crítica do artigo.
Referências

1. Lima-Costa MF, Barreto SM, Giatti L, Uchoa E. Situação socioeconômica e saúde entre brasileiros idosos em comparação aos mais jovens. In: Anais do I Workshop on Social and Gender Inequalities in Health among the Elderly in Brazil; 2002; Ouro Preto. p. 49-69.

2. Instituto Brasileiro de Geografia e Estatística (IBGE). Censo 2000. [site da Internet] [acessado $2010 \mathrm{mar}$ ]. Disponível em: http://www.ibge.gov.br/home/ estatistica/populacao/censo2000/default.shtm

3. Organização M undial da Saúde (OMS). Manual de vigilância das lesões. São Paulo: Secretaria de Estado da Saúde de São Paulo; 2004.

4. Departamento de Informática do Sistema Ú nico de Saúde (Datasus). M orbidade hospitalar do SUS: por local de residência - Brasil. [site da Internet] [acessado 2010 mar]. Disponível em: http://tabnet.datasus. gov.br/cgi/deftohtm.exe?sih/cnv/mrbr.def

5. Debert GG. A família e as novas políticas sociais no contexto brasileiro. Dossiê Comportamentos Familiares - Interseções 2001; 2:71-92.

6. Costa PL, Chaves PGS. A vivência afetiva e a violência doméstica contra os idosos. Belo Horizonte, 2003. (mimeo.)

7. Anetzberger GT, Korbin JE, Austin C. Alcoholism and elder abuse. Journal of Interpersonal Violence 1994; 9(2):184-193. 
8. M inayo M CS, Souza ER. As múltiplas mensagens da violência contra idosos. In: M inayo MCS, Souza $E R$, organizadores. Violência sob o olhar da saúde: a infrapolítica da contemporaneidade brasileira. Rio de Janeiro: Editora Fiocruz; 2003.

9. Faleiros VP. Violência contra a pessoa idosa: ocorrências, vítimas e agressores. Brasília: Universa; 2007.

10. M inayo MCS, Souza ER. Violência contra idosos: é possível prevenir? In: Brasil. Ministério da Saúde. Impacto da violência na saúde dos brasileiros. Brasília: Ministério da Saúde; 2005. p.141-165.

11. Abdala-Filho E. Avaliação de risco de violência em psiquiatria forense. Rev Psiq Clin 2004; 31(6):279284.

12. Reay AM, Browne KD. Risk factor characteristics in carers who physically abuse or neglect their elderly dependants. Aging M ental Helth 2001; 5(1):56-62.

13. Kleinschimidt KC. Elder abuse: a review. Annals of Emergency M edicine 1997; 30(4):463-472.

14. Souza ER, Ribeiro AP, Alice $S$, Souza AC, Marques CC. Rede de proteção aos idosos do Rio de Janeiro: um direito a ser conquistado. Cien Saude Colet 2008; 13(4):1153-1163.

15. Lancetti A, Amarante P. Saúde mental e saúde coletiva. In: Campos GW, M inayo MCS, Akerman M, Drumond Junior $M$, Carvalho $Y M$, organizadores. Tratado de saúde coletiva. São Paulo: Hucitec; Rio de Janeiro: Editora Fiocruz; 2006.

16. Kinoshita T. Saúde mental e antipsiquiatria em Santos: vinte anos depois. Cad Bras de Saude M ental 2009; 1(1):1-8

17. M inayo MCS, Souza ER. Violência e saúde como um campo interdisciplinar e de ação coletiva. História, Ciência, Saúde - M anguinhos 1997; 4(3):512-531.

18. Lopes CS, Faerstein E, Chor D. Eventos de vida produtores de estresse e transtornos mentais comuns: resultados do Estudo Pró-Saúde. Cad Saude Publica 2003; 19(6):1713-1720.

19. M artin D, Quirino J, M ari JJ. Depressão entre muIneres da periferia de São Paulo. Rev Saude Publica 2007; 41(4):591-597.

20. Santos AM CC. Articular saúde mental e relações de gênero: dar voz aos sujeitos silenciados. Cien Saude Colet 2009; 14(4):1177-1182.

21. Ximenes LF, Oliveira RVC, Assis SG. Violência e transtorno do estresse pós-traumático na infância. Cien Saude Colet 2009; 14(2):417-433.

22. Ribeiro WS, Andreoli SB, Ferri CP, Prince $M$, Mari JJ. Exposição à violência e problemas de saúde mental em países em desenvolvimento: uma revisão da literatura. Rev Bras Psiquiatr 2009; 31(supl II):S49-57.

23. Brasil. Lei no 8.842, de 4 de janeiro de 1994. Dispõe sobre a Política Nacional do Idoso, cria o Conselho Nacional do Idoso e dá outras providências. Brasília: [s.n]; 1994. [site da Internet] [acessado $2010 \mathrm{fev}$ 20]. Disponível em: http//www.sdee.aer.mil.br/ Legislacao/lei8842.pdf

24. Brasil. Estatuto do Idoso. Lei $n=10.741$, de 10 de outubro de 2003. Diário Oficial da União 2003; 3 out.

25. Brasil. M inistério da Saúde. Política de Saúde do Idoso. Portaria no 1.395, de 9 de dezembro de 1999 Brasília: M inistério da Saúde; 1999.
26. Brasil. M inistério da Saúde. Política Nacional de Redução da Morbimortalidade por Acidentes e Violências. Brasília: Ministério da Saúde; 2002.

27. Brasil. Portaria no 399, de 22 de fevereiro de 2006. Pacto pela Saúde. Braślia: M inistério da Saúde; 2006. [site da Internet] [acessado 2008 maio 3]. Disponível em: http://drt2001.saude.gov.br/sas/portarias

28. Souza ER. Análise diagnóstica de sistemas locais de saúde para atender aos agravos provocados por acidentes e violências contra idosos [relatório final de pesquisa] Rio de Janeiro: Claves/Fiocruz/CN Pq; 2008.

29. M inayo MCS, Sanchez O. Quantitativo-qualitativo: oposição ou complementaridade? Cad Saude Publica 1993; 9(3):239-262.

30. Varela J. Los métodos de consenso en el sector sanitário. Gaceta Sanitária 1991; 5:114-116.

31. Jones J, Hunter A. Consensus methods for medical and health services research. British M ed Journal 1995; 331:153-168.

32. Bardin L. Análise de conteúdo. Lisboa: Edições 70; 1979.

33. Leal EM, Delgado PGG. Clínica e cotidiano: o CAPS como dispositivo de desinstitucionalização. In: Pinheiro R, Guljor AP, Silva Júnior AGS, M attos RA, organizadores. Desinstitucionalização da saúde mental: contribuição para estudos avaliativos. Rio de Janeiro: Cepesc-IM S, Lappis-Abrasco; 2007.

34. Deslandes SF, Souza ER, M inayo MCS, Costa CRBSF, Krempel M, Cavalcanti ML, Lima M LC, Moysés S), Leal ML, Carmo CN. Diagnostic characterization of services providing care to victims of accidents and violence in five Brazilian state capitals. Cien Saude Colet 2006; 11(2):385-396.

35. Santos NC. Práticas sanitárias frente à violência intrafamiliar no âmbito do PSF: um estudo de caso. Rio de Janeiro: [s.n.]; 2005.

36. Guerra HL, Barreto SM, Uchoa E, Firmo JO, Costa MF. A morte dos idosos na Clínica Santa Genoveva, Rio de Janeiro: um excesso de mortalidade que o sistema público poderia ter evitado. Cad Saude Publica 2000; 16(12):545-551.

37. Souza ER, M inayo MCS, Ximenes LF, Deslandes SF. O idoso sob o olhar do outro. In: Minayo MCS, Coimbra Junior CE, organizadores. Antropologia, saúde e envelhecimento. Rio de Janeiro: Editora Fiocruz; 2002. p.191-209.

38. Dahlberg LL, Krug EG. Violência: um problema global de saúde pública. Cien Saude Colet 2006; 11(suppl):1163-1178. [site da Internet] [acessado 2010 fev 20]. Disponível em: http://www.scielo.br/ $\mathrm{pdf} / \mathrm{csc} / \mathrm{v} 11 \mathrm{~s} 0 / \mathrm{a} 07 \mathrm{v} 11 \mathrm{~s} 0$.pdf

Artigo apresentado em 23/02/2010

Aprovado em 02/05/2010

Versão final apresentada em 04/08/2010 